See discussions, stats, and author profiles for this publication at: https://www.researchgate.net/publication/282391634

\title{
Estimation of transmission line parameters using multiple methods
}

Article in IET Generation Transmission \& Distribution · September 2015

DOI: $10.1049 /$ iet-gtd.2014.0746

CITATIONS

7

2 authors:

Sergio Kurokawa

São Paulo State University

168 PUBLICATIONS 431 CITATIONS

SEE PROFILE

Some of the authors of this publication are also working on these related projects:

Project $\quad$ PMU for power distribution networks View project

Desenvolvimento de estudos em compostos de borracha de silicone com enfase em isolamento elétrico View project

\section{READS}

206

Eduardo C. M. Costa

University of São Paulo

71 PUBLICATIONS 202 CITATIONS

SEE PROFILE 


\title{
Estimation of transmission line parameters using multiple methods
}

ISSN 1751-8687

Received on 31st July 2014

Revised on 1st July 2015

Accepted on 7th August 2015

doi: 10.1049/iet-gtd.2014.0746 www.ietdl.org

\author{
Eduardo Coelho M. Costa ${ }^{\circledR}$, Sérgio Kurokawa ${ }^{2}$ \\ ${ }^{1}$ Departamento de Engenharia de Energia e Automação Elétricas - PEA, Escola Politécnica da Universidade de São Paulo - USP, \\ São Paulo, SP, Brazil \\ ${ }^{2}$ Departamento de Engenharia Elétrica - DEE, Faculdade de Engenharia de Ilha Solteira - FEIS, Universidade Estadual Paulista - UNESP, \\ Ilha Solteira, SP, Brazil \\ 凶E-mail: edumarquescosta@hotmail.com
}

\begin{abstract}
An estimation procedure for transmission line parameters is developed that combines a new method of determining line parameters with others available in the technical literature. Each estimation method has certain advantages and restrictions that depend on the operating conditions of the transmission system (e.g. the load profile), the physical characteristics of the line (e.g. the length and the geometry) and electromagnetic phenomena (e.g. singleor multiphase faults). The proposed estimation procedure exploits the major attributes of both the new determination method developed in this study and other well-established methods previously presented in the technical literature. Based on current and voltage measurements obtained from synchronised fault records from both line terminals, a combination of multiple methods can be used to accurately estimate most line parameters, a task that is typically impossible using a single method.
\end{abstract}

\section{Introduction}

Knowledge of the electrical characteristics of a power transmission system is an important requirement for reliable operation of an electric power system. Accurate parameter determination in a power grid is directly related to many aspects of power system analysis such as fault detection/location in overhead transmission lines and underground cables, correct parameterisation of protection systems, proper analysis of the insulation coordination, and knowledge of the propagation characteristics, transient conditions and possible overvoltages in the transmission system, which is necessary for the design of the surge protection system.

The electrical parameters of transmission lines can be calculated as a function of the line structure and the physical characteristics of the lines (e.g. the line height, the geometry of the towers and phases, the soil characteristics and the cable properties) while considering the earth-return current and the skin effect in the wires. Determining line parameters using the classical analytical methods based on Bessel and Carson functions results in certain inaccuracies because of the approximations that are used for the varying environmental conditions, the non-homogeneous soil conductivity and the geometrical structure of the lines [1,2].

Line parameters can also be estimated based on current and voltage measurements at the line terminals. These measurements can be obtained in a synchronised manner using phasor measurement units (PMUs) or using fault records obtained from protective relays at the sending and receiving ends of the transmission lines [3-7]. Theoretically, the estimation of the parameters of transmission lines from such synchronised measurements is a simple and accurate procedure; however, from a practical standpoint and based on the few methods available in the technical literature, line parameter estimation is far from being a trivial task. The performance of the various estimation methods differs depending on the system characteristics, the load profile, the line geometry and the system dynamics (i.e. transient or steady state). Therefore, each estimation method is effective for specific operating conditions of the power system, and typically, only a few parameters of the impedance and admittance matrices, $[\boldsymbol{Z}]$ and $[\boldsymbol{Y}]$, respectively, can be accurately estimated $[5,8]$.
The estimation methods that are available in the technical literature may be classified into two basic types: frequency- and time-domain methods [5, 8]. Most frequency-domain methods were developed for transposed or symmetrical transmission lines with a vertical plane of symmetry. In these methods, the line parameters are estimated based on synchronised measurements of current and voltage phasors at both line terminals [5, 6]. However, frequency-domain methods have been shown to be unreliable for certain line configurations and operating conditions. For example, the frequency-domain method for symmetrical lines proposed in [3] is not fully reliable because the filter-based phasor calculation may cause errors in the parameter determination [4]. Kurokawa et al. [5] present an estimation method that identifies the resistance parameters of three-phase transmission lines with reasonable accuracy; however, the accuracy depends on the line structure (the geometrical characteristics) and the load profile at the receiving end of the line. Meanwhile, the estimation method proposed in [6] is restricted to determining the inductance parameters of transmission lines. Furthermore, $\mathrm{Hu}$ and $\mathrm{Chen} \mathrm{[6]} \mathrm{provide} \mathrm{an}$ interesting discussion of some of the possible limitations of parameter estimation using frequency-domain methods and synchronised phasor measurements.

There are also frequency-domain estimation methods based on unsynchronised phasor measurements and post-synchronisation methods. In [8], the Prony method is used to overcome the inaccuracies introduced by the re-synchronisation of fault data. These inaccuracies are partially resolved by constructing the fault signal from the fault records using digital signal-processing techniques.

A time-domain estimation method based on the representation of a line by a three-phase $\pi$ circuit using a temporal sequence has been proposed [4]. The differential equations for the equivalent three-phase $\pi$ circuit are expressed as state equations, and the parameter estimates are obtained using time-domain fault records obtained from protective relays. The first-order system consists of a set of differential equations (state equations), where the number of equations depends on the number of samples extracted from the fault records. The solution of the state equations is obtained using the well-established least-squares method. Theoretically, the 
time-domain method proposed in [4] is capable of identifying most of the parameters in $[\boldsymbol{Z}]$ and $[\boldsymbol{Y}]$, but there are certain restrictions. First, the method applies only to a single-phase fault, where only the resistance and the inductance of the faulty phase can be determined with good accuracy. In addition, the mutual parameters in $[\boldsymbol{Z}]$ and $[\boldsymbol{Y}]$ cannot be estimated with acceptable accuracy. The Prony method can be used to mitigate these errors that arise in the time-domain method proposed in [4] by fitting the fault signal obtained from the fault records. Using this method, which is based on digital signal processing, the time-domain method of [4] can be used to estimate other line parameters with acceptable accuracy. However, the application of the Prony method for parameter estimation requires a very complex algorithm. Although the estimation procedure using the Prony method can determine the line parameters with acceptable accuracy, from a practical standpoint, it is desirable to considerably simplify the estimation process.

This research investigates a simpler alternative estimation procedure in the time domain that combines two different methods. One of these methods is essentially the time-domain method developed in [4] without the use of the Prony method. This method performs well in the determination of the self-resistance and self-inductance of a faulty phase. The transmission line is represented by a three-phase $\pi$ circuit, where the impedance and admittance parameters in the matrices $[\boldsymbol{Z}]$ and $[\boldsymbol{Y}]$, respectively, are intrinsically represented by electrical circuit elements. As the line model presented in [4] is developed directly from the $\boldsymbol{Z}$ and $\boldsymbol{Y}$ parameters of the line, it is a phase-domain model. The other estimation method that is used is a new procedure based on modal decoupling techniques, i.e. the three-phase transmission line is decoupled into three independent propagation modes, which are modelled as three single-phase lines using conventional $\pi$ circuits, unlike the three-phase $\pi$ representation required in the first method. Thus, this estimation method exists in the modal domain.

The premise of this research is that the use of two or more estimation methods provides redundancy, i.e. greater confidence in the estimates. Thus, using a large database and suitable analysis, more reliable values of $\boldsymbol{Z}$ and $\boldsymbol{Y}$ parameters can be estimated.

\section{Transmission line electrical parameters}

The propagation characteristics of multiconductor transmission lines are defined by the electrical parameters in the impedance and admittance matrices, $[\boldsymbol{Z}]$ and $[\boldsymbol{Y}]$, respectively. These matrices are expressed as in (1) and (2).

$$
\begin{array}{r}
{[\boldsymbol{Z}]=\left[\begin{array}{cccc}
Z_{11} & Z_{12} & \cdots & Z_{1 n} \\
Z_{21} & Z_{22} & \cdots & Z_{2 n} \\
\vdots & \vdots & \ddots & \vdots \\
Z_{n 1} & Z_{n 2} & \cdots & Z_{n n}
\end{array}\right]} \\
{[\boldsymbol{Y}]=\left[\begin{array}{cccc}
Y_{11} & Y_{12} & \cdots & Y_{1 n} \\
Y_{21} & Y_{22} & \cdots & Y_{2 n} \\
\vdots & \vdots & \ddots & \vdots \\
Y_{n 1} & Y_{n 2} & \cdots & Y_{n n}
\end{array}\right]}
\end{array}
$$

Matrices $[\boldsymbol{Z}]$ and $[\boldsymbol{Y}]$ are square matrices with dimensions equal to the number of phases $n$. The terms on the main diagonals are the self-impedances or self-admittances, and the remaining terms are the mutual impedances or admittances, which represent the interactions between phases. The self- and mutual impedances in $[Z$ consist of a frequency-dependent resistance and a frequency-dependent inductance.

The self-impedances consist of a real component that represents the frequency-dependent resistance and an imaginary term associated with the frequency-dependent inductance. The self-impedance depends on the skin effect in the wires, the earth-return current through the soil, and a constant term that represents the external impedance. The mutual impedance terms in the impedance matrix $[Z]$ are functions of the earth-return impedance and the external impedance related to the electromagnetic coupling between the two phases [9].

Conventionally, the skin effect is calculated using Bessel functions and the physical and geometrical characteristics of the conductors [2]. The earth-return impedance is calculated using the Carson trigonometric series, assuming a constant value for the soil conductivity [1]. This assumption may be inappropriate because the soil conductivity is highly variable, depending on geological formations and environmental conditions. The external impedance is constant, and it is calculated based on the geometrical and structural characteristics of the line [9].

The impedance matrix $[\boldsymbol{Z}]$ is composed of a resistance matrix $[\boldsymbol{R}]$ and an inductance matrix $[\boldsymbol{L}]$ which can be expressed as follows for an $n$-phase line

$$
\begin{gathered}
{[\boldsymbol{R}]=\left[\begin{array}{cccc}
R_{11} & R_{12} & \cdots & R_{1 n} \\
R_{21} & R_{22} & \cdots & R_{2 n} \\
\vdots & \vdots & \ddots & \vdots \\
R_{n 1} & R_{n 2} & \cdots & R_{n n}
\end{array}\right]} \\
{[\boldsymbol{L}]=\left[\begin{array}{cccc}
L_{11} & L_{12} & \cdots & L_{1 n} \\
L_{21} & L_{22} & \cdots & L_{2 n} \\
\vdots & \vdots & \ddots & \vdots \\
L_{n 1} & L_{n 2} & \cdots & L_{n n}
\end{array}\right]}
\end{gathered}
$$

The admittance matrix $[\boldsymbol{Y}]$ is composed of conductance and capacitance terms. Conventionally, in transmission line modelling (TLM), the transverse conductance is neglected in the admittance calculation. Thus, the real term of the complex admittance is neglected, and $\boldsymbol{Y}$ becomes a purely imaginary value representing a capacitive reactance [9].

The self- and mutual capacitances in $[\boldsymbol{Y}]$ do not depend on the frequency and are calculated as functions of the geometrical and physical characteristics of the line and the conductivity of the air. This approach may also be inappropriate because the shunt capacitance and the capacitances between phases are intrinsically associated with environmental conditions such as the atmospheric humidity, the dielectric constant and the weather [10]. The capacitance matrix for a multiconductor transmission line can be expressed as follows

$$
[\boldsymbol{C}]=\left[\begin{array}{cccc}
C_{11} & C_{12} & \cdots & C_{1 n} \\
C_{21} & C_{22} & \cdots & C_{2 n} \\
\vdots & \vdots & \ddots & \vdots \\
C_{n 1} & C_{n 2} & \cdots & C_{n n}
\end{array}\right]
$$

The preceding discussion of transmission line parameters demonstrates that the electrical parameters can be calculated using several approaches and mathematical tools. However, the $\boldsymbol{R}, \boldsymbol{L}$ and $\boldsymbol{C}$ parameters can be obtained with greater accuracy using the estimation methods that are discussed in the following sections.

\section{Parameter estimation using combined methods}

The estimation procedure developed in this research combines the results obtained from two different and independent estimation methods and uses current and voltage measurements collected by protective relays at the receiving and sending ends of the line during a fault.

The primary difference between the two estimation methods lies in the line modelling. In the first estimation method, the transmission line is represented as a three-phase $\pi$ circuit, in which the self- and mutual line parameters in $[\boldsymbol{Z}]$ and $[\boldsymbol{Y}]$ are modelled as electric circuit elements. Thus, this line representation is characterised as a phase-domain model because the impedance and 
admittance parameters are modelled directly from the line parameters contained in $[\boldsymbol{Z}]$ and $[\boldsymbol{Y}]$. By contrast, the second estimation method is based on modal decoupling techniques, i.e. an $n$-phase transmission line is decoupled into $n$-independent propagation modes. Hence, there are $n$-independent systems that can be represented as $n$ single-phase lines. Therefore, the line model that is used in the second method is a conventional $\pi$ circuit, which has been well studied in the literature on power systems. As the second estimation method is based on the individual modelling of each propagation mode, this method exists in the modal domain. Both estimation methods are applied to the same current and voltage data recorded at the line terminals during a single-phase fault, which can be simulated using software such as the electromagnetic transient program (EMTP) [11].

The combination of two different estimation methods provides redundancy in the results. Both estimation methods are used to estimate the electrical parameters of the same transmission line based on the same current and voltage measurements. Thus, using a redundant database and suitable analysis, more reliable values of the $\boldsymbol{R}, \boldsymbol{L}$ and $\boldsymbol{C}$ parameters can be estimated.

\subsection{Parameter determination based on the phase-domain line model}

The estimation method that is performed using a phase-domain line model is based on the representation of the transmission line using a three-phase $\pi$ circuit, as illustrated in Fig. 1.

The line parameters in $[\boldsymbol{Z}]$ and $[\boldsymbol{Y}]$ are represented by lumped $\boldsymbol{R}, \boldsymbol{L}$ and $\boldsymbol{C}$ elements. The electrical circuit depicted in Fig. 1 is an equivalent representation of a three-phase transmission line [4].

Fig. 1 shows the phase voltages at the sending and receiving ends of the line, which are labelled as terminals $A$ and $B$, respectively. The voltages at terminal $A$ for phases 1,2 and 3 are $v_{A 1}, v_{A 2}$ and $v_{A 3}$, respectively, and are expressed in vector form in (6). For terminal $B$, the phase voltages $v_{B 1}, v_{B 2}$ and $v_{B 3}$ are analogously expressed in (7). The currents at terminal $A$ are $i_{A 1}, i_{A 2}$ and $i_{A 3}$, and the currents at terminal $B$ are $i_{B 1}, i_{B 2}$ and $i_{B 3}$; these are expressed in vector form in (8) and (9), respectively. There are $\boldsymbol{p}$ measurements of current and voltage, which are obtained from the fault data recorded at both line terminals, such that in the equations below, $m=1,2, \ldots, \boldsymbol{p}$. Hence, there are $\boldsymbol{p}$ vectors $\left[\boldsymbol{v}_{A}\right],\left[\boldsymbol{v}_{B}\right],\left[\boldsymbol{i}_{A}\right]$ and $\left[\boldsymbol{i}_{B}\right]$, one for each measurement/record of voltage and current at the line terminals. Previous knowledge (i.e. measurements) of the currents and voltages is required for the parameter estimation [4].

$$
\begin{aligned}
{\left[v_{A}\right]_{m} } & =\left[\begin{array}{lll}
v_{A 1} & v_{A 2} & v_{A 3}
\end{array}\right]^{\mathrm{T}} \\
{\left[v_{B}\right]_{m} } & =\left[\begin{array}{lll}
v_{B 1} & v_{B 2} & v_{B 3}
\end{array}\right]^{\mathrm{T}} \\
{\left[i_{A}\right]_{m} } & =\left[\begin{array}{lll}
i_{A 1} & i_{A 2} & i_{A 3}
\end{array}\right]^{\mathrm{T}} \\
{\left[i_{B}\right]_{m} } & =\left[\begin{array}{lll}
i_{B 1} & i_{B 2} & i_{B 3}
\end{array}\right]^{\mathrm{T}}
\end{aligned}
$$

The superscript $\mathrm{T}$ indicates the transpose of a vector in (6)-(9) above.

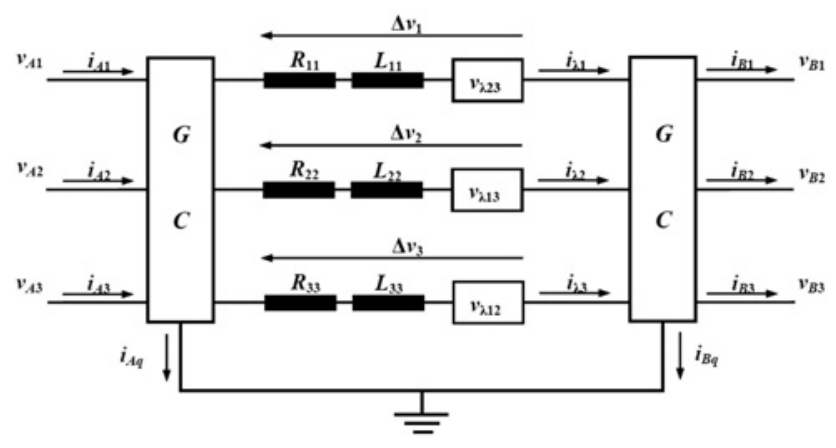

Fig. 1 Transmission line represented by a three-phase $\pi$ circuit
The self-resistances and -inductances are explicitly indicated in Fig. 1, whereas the mutual parameters are implicitly represented in the mutual voltages induced in phases 1,2 and 3 , namely, $v_{\lambda 23}$, $v_{\lambda 13}$ and $v_{\lambda 12}$, respectively. The transverse conductances $\boldsymbol{G}$ and the transverse capacitances $\boldsymbol{C}$ are also implicit in Fig. 1. The terms $i_{A q}$ and $i_{B q}$ are the shunt currents through the shunt admittances of phases 1, 2 and 3, as expressed in (10) and (11) [4].

$$
\begin{aligned}
& {\left[i_{A q}\right]_{m}=\left[\begin{array}{lll}
i_{A q 1} & i_{A q 2} & i_{A q 3}
\end{array}\right]^{\mathrm{T}}} \\
& {\left[i_{B q}\right]_{m}=\left[\begin{array}{lll}
i_{B q 1} & i_{B q 2} & i_{B q 3}
\end{array}\right]^{\mathrm{T}}}
\end{aligned}
$$

The unknown currents through the longitudinal self-resistances $\boldsymbol{R}$ and the longitudinal inductances $\boldsymbol{L}$ are denoted by $i_{\lambda 1}, i_{\lambda 2}$ and $i_{\lambda 3}$. Thus, the voltage drop along the line is expressed by the following system of differential equations [4]

$$
[\Delta v]_{m}=[\boldsymbol{R}]\left[i_{\lambda}\right]_{m}+[\boldsymbol{L}] \frac{\mathrm{d}}{\mathrm{d} t}\left[i_{\lambda}\right]_{m}
$$

where $[\Delta v]_{m}$ and $\left[i_{\lambda}\right]_{m}$ are expressed as in (13) and (14).

$$
\begin{aligned}
{[\Delta v]_{m} } & =\left[\begin{array}{lll}
\Delta v_{1} & \Delta v_{2} & \Delta v_{3}
\end{array}\right]^{\mathrm{T}} \\
{\left[i_{\lambda}\right]_{m} } & =\left[\begin{array}{lll}
i_{\lambda 1} & i_{\lambda 2} & i_{\lambda 3}
\end{array}\right]^{\mathrm{T}}
\end{aligned}
$$

The shunt admittances connected at terminals $A$ and $B$ are determined from the shunt currents expressed in (10) and (11) and from the voltage records obtained at both line terminals [4]

$$
\begin{aligned}
& {\left[i_{A q}\right]_{m}=[\boldsymbol{G}]\left[v_{A}\right]_{m}+[\boldsymbol{C}] \frac{\mathrm{d}}{\mathrm{d} t}\left[v_{A}\right]_{m}} \\
& {\left[i_{B q}\right]_{m}=[\boldsymbol{G}]\left[v_{B}\right]_{m}+[\boldsymbol{C}] \frac{\mathrm{d}}{\mathrm{d} t}\left[v_{B}\right]_{m}}
\end{aligned}
$$

If the conductance matrix $[\boldsymbol{G}]$ is neglected, then the shunt currents $i_{A q}$ and $i_{B q}$ become functions of $\mathrm{d} v_{A} / \mathrm{d} t$ and $\mathrm{d} v_{B} / \mathrm{d} t$, respectively.

Therefore, given the voltages $\left[v_{A}\right]_{m}$ and $\left[v_{B}\right]_{m}$ and the currents $\left[i_{A}\right]_{m}$ and $\left[i_{B}\right]_{m}$ for $\boldsymbol{p}$ samples obtained from the fault records, the system of differential equations obtained from (12), (15) and (16) can be solved using the least-squares method. The step-by-step algorithm for the phase-domain estimation method is as follows:

(a) Calculate $\left[v_{A}\right]_{m},\left[v_{B}\right]_{m},\left[i_{A}\right]_{m}$ and $\left[i_{B}\right]_{m}$ in (6)-(9) from the fault records, where $m=1,2, \ldots, \boldsymbol{p}$ is the index representing the current and voltage samples recorded during the fault;

(b) Calculate $[\Delta v]_{m}$ and $\left[i_{\lambda}\right]_{m}$ based on the electric circuit representation depicted in Fig. 1 and the known values of the voltages and currents at the line terminals [4];

(c) Form the system of differential equations based on (12), (15) and (16);

(d) Solve the system of differential equations using the least-squares method [4];

(e) $\boldsymbol{R}, \boldsymbol{L}$ and $\boldsymbol{C}$ parameters in (3)-(5) are estimated from the solution of (15) and (16).

\subsection{Parameter determination based on a modal-domain line model}

The second method used in the combined estimation procedure is based on a line model using modal decoupling techniques and a lumped-parameter representation. A similar line model has been used in previous research to study electromagnetic transients in power transmission systems $[12,13]$. However, a modified version of this line model is developed in this section for the determination of line parameters at an industrial frequency.

The estimation method is developed in the modal domain, where the three phases are decoupled into three independent propagation 
modes that are modelled as three single-phase lines. Each propagation mode is modelled as a single $\pi$ circuit in which the mutual parameters are neglected. The system of differential equations that is obtained from the line representation with lumped parameters is solved using the least-squares method.

Initially, $\boldsymbol{p}$ samples of voltage and current are obtained from the fault data recorded at both line terminals, as described in (6)-(9). As $\boldsymbol{p}$ vectors of voltage and current are known, the modal voltages and currents are calculated using a constant and real transformation matrix $[\boldsymbol{T}][14]$

$$
\begin{aligned}
& {\left[v_{A \mathrm{M}}\right]_{m}=[\boldsymbol{T}]^{\mathrm{T}}\left[v_{A}\right]_{m}} \\
& {\left[v_{B \mathrm{M}}\right]_{m}=[\boldsymbol{T}]^{\mathrm{T}}\left[v_{B}\right]_{m}} \\
& {\left[i_{A \mathrm{M}}\right]_{m}=[\boldsymbol{T}]^{-1}\left[i_{A}\right]_{m}} \\
& {\left[i_{B \mathrm{M}}\right]_{m}=[\boldsymbol{T}]^{-1}\left[i_{B}\right]_{m}}
\end{aligned}
$$

The matrix $[\boldsymbol{T}]$ is obtained from the product $[\boldsymbol{Z}][\boldsymbol{Y}]$ or $[\boldsymbol{Y}][\boldsymbol{Z}]$, where the rows and columns of $[\boldsymbol{T}]$ are functions of the eigenvalues and eigenvectors of the resulting matrix product. A real-valued and constant transformation matrix is possible for transmission lines with a vertical plane of symmetry or nearly symmetric matrices $[\boldsymbol{Z}]$ and $[\boldsymbol{Y}]$. If $[\boldsymbol{Z}]$ and $[\boldsymbol{Y}]$ are approximately symmetric, then the transformation matrix contains real and constant terms [13]. Thus, the transformation matrix $[\boldsymbol{T}]$ considered in the successive modal transformations is the well-known Clarke's matrix, since the parameters are unknown.

From (17)-(20), the voltage and current vectors are calculated for three independent propagation modes and $m=1,2, \ldots, \boldsymbol{p}$.

$$
\begin{gathered}
{\left[v_{A \mathrm{M}}\right]_{m}=\left[\begin{array}{lll}
v_{A \alpha} & v_{A \beta} & v_{A 0}
\end{array}\right]^{\mathrm{T}}} \\
{\left[v_{B \mathrm{M}}\right]_{m}=\left[\begin{array}{lll}
v_{B \alpha} & v_{B \beta} & v_{B 0}
\end{array}\right]^{\mathrm{T}}} \\
{\left[i_{A \mathrm{M}}\right]_{m}=\left[\begin{array}{lll}
i_{A \alpha} & i_{A \beta} & i_{A 0}
\end{array}\right]^{\mathrm{T}} ; \quad\left[i_{B \mathrm{M}}\right]_{m}=\left[\begin{array}{lll}
i_{B \alpha} & i_{B \beta} & i_{B 0}
\end{array}\right]^{\mathrm{T}}}
\end{gathered}
$$

In (21) and (22), the voltages and currents are obtained for the propagation modes $\alpha, \beta$ and 0 . Thus, considering that the voltages and currents are known at both terminals for the propagation modes $\alpha, \beta$ and 0 , the $\boldsymbol{R}, \boldsymbol{L}$ and $\boldsymbol{C}$ matrices in the modal domain are expressed as follows

$$
\begin{aligned}
& {\left[\boldsymbol{R}_{\mathrm{M}}\right]=\left[\begin{array}{ccc}
R_{\alpha} & 0 & 0 \\
0 & R_{\beta} & 0 \\
0 & 0 & R_{0}
\end{array}\right]} \\
& {\left[\boldsymbol{L}_{\mathrm{M}}\right]=\left[\begin{array}{ccc}
L_{\alpha} & 0 & 0 \\
0 & L_{\beta} & 0 \\
0 & 0 & L_{0}
\end{array}\right]} \\
& {\left[\boldsymbol{C}_{\mathrm{M}}\right]=\left[\begin{array}{ccc}
C_{\alpha} & 0 & 0 \\
0 & C_{\beta} & 0 \\
0 & 0 & C_{0}
\end{array}\right]}
\end{aligned}
$$

In (23)-(25), the modal-domain parameters are represented by $\left[\boldsymbol{R}_{\mathrm{M}}\right]$, $\left[\boldsymbol{L}_{\mathrm{M}}\right]$ and $\left[\boldsymbol{C}_{\mathrm{M}}\right]$, where the subscript $\mathrm{M}$ denotes the mode, either $\alpha, \beta$ or 0 . The off-diagonal elements of $\left[\boldsymbol{R}_{\mathrm{M}}\right],\left[\boldsymbol{L}_{\mathrm{M}}\right]$ and $\left[\boldsymbol{C}_{\mathrm{M}}\right]$ are zero, i.e. there are no mutual terms, which indicate that there is no coupling among the modes [13].

As the electrical parameters can be described in the modal domain, the propagation modes can be modelled as three single-phase lines using a single $\pi$ circuit, as shown in Fig. 2.

The system of differential equations that represents the equivalent circuit depicted in Fig. 2 is expressed in the modal domain as follows

$$
\left[\Delta v_{\mathrm{M}}\right]_{m}=\left[\boldsymbol{R}_{\mathrm{M}}\right]\left[i_{\lambda \mathrm{M}}\right]_{m}+\left[\boldsymbol{L}_{\mathrm{M}}\right] \frac{\mathrm{d}}{\mathrm{d} t}\left[i_{\lambda \mathrm{M}}\right]_{m}
$$

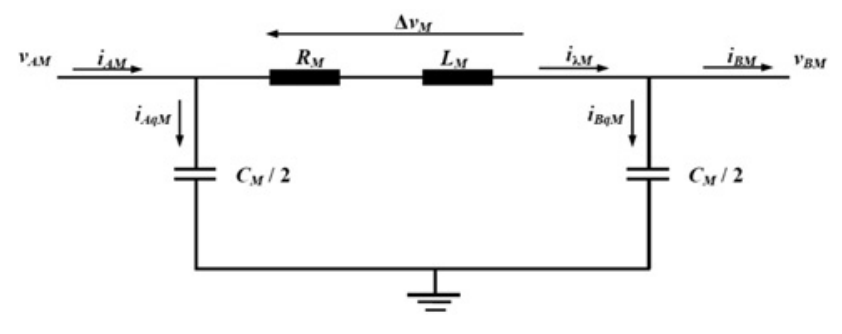

Fig. 2 Propagation mode modelled using a single $\pi$ circuit

where $\left[\Delta v_{\mathrm{M}}\right]_{m}$ and $\left[i_{\lambda \mathrm{M}}\right]_{m}$ are vectors that contain the voltage drops over the modal longitudinal parameters and the currents through the modal resistances, respectively, with $m=1,2, \ldots, \boldsymbol{p}$

$$
\begin{aligned}
{\left[\Delta v_{\mathrm{M}}\right]_{m} } & =\left[\begin{array}{lll}
\Delta v_{\alpha} & \Delta v_{\beta} & \Delta v_{0}
\end{array}\right]^{\mathrm{T}} \\
{\left[i_{\lambda \mathrm{M}}\right]_{m} } & =\left[\begin{array}{lll}
i_{\lambda \alpha} & i_{\lambda \beta} & i_{\lambda 0}
\end{array}\right]^{\mathrm{T}}
\end{aligned}
$$

Based on the $\pi$ representation illustrated in Fig. 2, the capacitances of modes $\alpha, \beta$ and 0 are obtained from the differential equations defined in (29) and (30) at terminals $A$ and $B$, respectively

$$
\begin{aligned}
& {\left[i_{A q \mathrm{M}}\right]_{m}=\frac{\left[\boldsymbol{C}_{\mathrm{M}}\right]}{2} \frac{\mathrm{d}}{\mathrm{d} t}\left[v_{A \mathrm{M}}\right]_{m}} \\
& {\left[i_{B q \mathrm{M}}\right]_{m}=\frac{\left[\boldsymbol{C}_{\mathrm{M}}\right]}{2} \frac{\mathrm{d}}{\mathrm{d} t}\left[v_{B \mathrm{M}}\right]_{m}}
\end{aligned}
$$

As previously noted, the $\boldsymbol{G}$ parameters are typically neglected in TLM [9]. Thus, the currents through terminals $A$ and $B$ can be expressed as functions of $\boldsymbol{C}_{\mathrm{M}}$, as shown in Fig. 2 and in (29) and (30).

The vectors for the modal shunt currents $\left[i_{A q \mathrm{M}}\right]$ and $\left[i_{B q \mathrm{M}}\right]$ are expressed in the transposed form as follows

$$
\begin{aligned}
& {\left[i_{A q \mathrm{M}}\right]_{m}=\left[\begin{array}{lll}
i_{A q \alpha} & i_{A q \beta} & i_{A q 0}
\end{array}\right]^{\mathrm{T}}} \\
& {\left[i_{B q \mathrm{M}}\right]_{m}=\left[\begin{array}{lll}
i_{B q \alpha} & i_{B q \beta} & i_{B q 0}
\end{array}\right]^{\mathrm{T}}}
\end{aligned}
$$

Through substitution of (30) into (29) and algebraic manipulations, $\left[i_{A q \mathrm{M}}\right]$ can be expressed individually for each propagation mode as a function of $\left[i_{B q \mathrm{M}}\right],\left[v_{A q \mathrm{M}}\right]$ and $\left[v_{B \mathrm{M}}\right]$ :

$$
\begin{gathered}
{\left[i_{A q \alpha}\right]=\left[i_{B q \alpha}\right]\left[\dot{v}_{B \alpha}\right]^{\mathrm{T}}\left(\left[\dot{v}_{B \alpha}\right]\left[\dot{v}_{B \alpha}\right]^{\mathrm{T}}\right)^{-1}\left[\dot{v}_{A \alpha}\right]=\left[i_{B q \alpha}\right]\left[\boldsymbol{Q}_{\alpha}\right]} \\
{\left[i_{A q \beta}\right]=\left[i_{B q \beta}\right]\left[\dot{v}_{B \beta}\right]^{\mathrm{T}}\left(\left[\dot{v}_{B \beta}\right]\left[\dot{v}_{B \beta}\right]^{\mathrm{T}}\right)^{-1}\left[\dot{v}_{A \beta}\right]=\left[i_{B q \beta}\right]\left[\boldsymbol{Q}_{\beta}\right]} \\
{\left[i_{A q 0}\right]=\left[i_{B q 0}\right]\left[\dot{v}_{B 0}\right]^{\mathrm{T}}\left(\left[\dot{v}_{B 0}\right]\left[\dot{v}_{B 0}\right]^{\mathrm{T}}\right)^{-1}\left[\dot{v}_{A 0}\right]=\left[i_{B q 0}\right]\left[\boldsymbol{Q}_{0}\right]}
\end{gathered}
$$

where the matrices $\left[\boldsymbol{Q}_{\alpha}\right],\left[\boldsymbol{Q}_{\beta}\right]$ and $\left[\boldsymbol{Q}_{0}\right]$ are expressed as follows

$$
\begin{gathered}
{\left[\boldsymbol{Q}_{\alpha}\right]=\left[\dot{v}_{B \alpha}\right]^{\mathrm{T}}\left(\left[\dot{v}_{B \alpha}\right]\left[\dot{v}_{B \alpha}\right]^{T}\right)^{-1}\left[\dot{v}_{A \alpha}\right]} \\
{\left[\boldsymbol{Q}_{\beta}\right]=\left[\dot{v}_{B \beta}\right]^{\mathrm{T}}\left(\left[\dot{v}_{B \beta}\right]\left[\dot{v}_{B \beta}\right]^{\mathrm{T}}\right)^{-1}\left[\dot{v}_{A \beta}\right]} \\
{\left[\boldsymbol{Q}_{0}\right]=\left[\dot{v}_{B 0}\right]^{\mathrm{T}}\left(\left[\dot{v}_{B 0}\right]\left[\dot{v}_{B 0}\right]^{\mathrm{T}}\right)^{-1}\left[\dot{v}_{A 0}\right]}
\end{gathered}
$$

The matrices of the derivatives of the modal voltages at terminals $A$ 
and $B$ for $\alpha, \beta$ and 0 , for $p$ fault records, are expressed as follows

$$
\begin{gathered}
{\left[\dot{v}_{A \alpha}\right]=\left[\begin{array}{llll}
\frac{\mathrm{d} v_{A \alpha, 1}}{\mathrm{~d} t} & \frac{\mathrm{d} v_{A \alpha, 2}}{\mathrm{~d} t} & \cdots & \frac{\mathrm{d} v_{A \alpha, p}}{\mathrm{~d} t}
\end{array}\right] ;} \\
{\left[\dot{v}_{B \alpha}\right]=\left[\begin{array}{llll}
\frac{\mathrm{d} v_{B \alpha, 1}}{\mathrm{~d} t} & \frac{\mathrm{d} v_{B \alpha, 2}}{\mathrm{~d} t} & \cdots & \frac{\mathrm{d} v_{B \alpha, p}}{\mathrm{~d} t}
\end{array}\right]} \\
{\left[\dot{v}_{A \beta}\right]=\left[\begin{array}{llll}
\frac{\mathrm{d} v_{A \beta, 1}}{\mathrm{~d} t} & \frac{\mathrm{d} v_{A \beta, 2}}{\mathrm{~d} t} & \cdots & \frac{\mathrm{d} v_{A \alpha, p}}{\mathrm{~d} t}
\end{array}\right] ;} \\
{\left[\dot{v}_{B \beta}\right]=\left[\begin{array}{llll}
\frac{\mathrm{d} v_{B \beta, 1}}{\mathrm{~d} t} & \frac{\mathrm{d} v_{B \beta, 2}}{\mathrm{~d} t} & \cdots & \frac{\mathrm{d} v_{B \beta, p}}{\mathrm{~d} t}
\end{array}\right]} \\
{\left[\dot{v}_{A 0}\right]=\left[\begin{array}{llll}
\frac{\mathrm{d} v_{A 0,1}}{\mathrm{~d} t} & \frac{\mathrm{d} v_{A 0,2}}{\mathrm{~d} t} & \cdots & \frac{\mathrm{d} v_{A 0, p}}{\mathrm{~d} t}
\end{array}\right] ;} \\
{\left[\dot{v}_{B 0}\right]=\left[\begin{array}{llll}
\frac{\mathrm{d} v_{B 0,1}}{\mathrm{~d} t} & \frac{\mathrm{d} v_{B 0,2}}{\mathrm{~d} t} & \cdots & \frac{\mathrm{d} v_{B 0, p}}{\mathrm{~d} t}
\end{array}\right]}
\end{gathered}
$$

The shunt currents at terminals $A$ and $B$ for the propagation modes $\alpha$, $\beta$ and 0 , are expressed for $p$ fault samples as

$$
\begin{gathered}
{\left[i_{A q \alpha}\right]=\left[\begin{array}{llll}
i_{A q \alpha, 1} & i_{A q \alpha, 2} & \cdots & i_{A q \alpha, p}
\end{array}\right] ;} \\
{\left[i_{B q \alpha}\right]=\left[\begin{array}{llll}
i_{B q \alpha, 1} & i_{B q \alpha, 2} & \cdots & i_{B q \alpha, p}
\end{array}\right]} \\
{\left[i_{A q \beta}\right]=\left[\begin{array}{llll}
i_{A q \beta, 1} & i_{A q \beta, 2} & \cdots & i_{A q \beta, p}
\end{array}\right] ;} \\
{\left[i_{B q \beta}\right]=\left[\begin{array}{llll}
i_{B q \beta, 1} & i_{B q \beta, 2} & \cdots & i_{B q \beta, p}
\end{array}\right]} \\
{\left[i_{A q 0}\right]=\left[\begin{array}{llll}
i_{A q 0,1} & i_{A q 0,2} & \cdots & i_{A q 0, p}
\end{array}\right] ;} \\
{\left[i_{B q 0}\right]=\left[\begin{array}{llll}
i_{B q 0,1} & i_{B q 0,2} & \cdots & i_{B q 0, p}
\end{array}\right]}
\end{gathered}
$$

From the $\pi$ circuit depicted in Fig. 2, the unknown currents in the vectors $\left[\boldsymbol{i}_{\lambda \alpha}\right],\left[\boldsymbol{i}_{\lambda \beta}\right]$ and $\left[\boldsymbol{i}_{\lambda 0}\right]$ are obtained as

$$
\begin{aligned}
& {\left[\boldsymbol{i}_{\lambda \alpha}\right]=\left[i_{A \alpha}\right]-\left[i_{A q \alpha}\right]=\left[i_{B \alpha}\right]+\left[i_{B q \alpha}\right]} \\
& {\left[\boldsymbol{i}_{\lambda \beta}\right]=\left[i_{A \beta}\right]-\left[i_{A q \beta}\right]=\left[i_{B \beta}\right]+\left[i_{B q \beta}\right]} \\
& {\left[\boldsymbol{i}_{\lambda 0}\right]=\left[i_{A 0}\right]-\left[i_{A q 0}\right]=\left[i_{B 0}\right]+\left[i_{B q 0}\right]}
\end{aligned}
$$

By substituting $\left[i_{B q \alpha}\right],\left[i_{B q \beta}\right]$ and $\left[i_{B q 0}\right]$ into (45)-(47), the current vectors $\left[\boldsymbol{i}_{A q \alpha}\right],\left[\boldsymbol{i}_{A q \beta}\right]$ and $\left[\boldsymbol{i}_{A q 0}\right]$ can be reformulated as functions of the current and voltage data at terminals $A$ and $B$ of $\alpha, \beta$ and 0

$$
\begin{gathered}
{\left[\boldsymbol{i}_{A q \alpha}\right]=\left(\left[i_{A \alpha}\right]-\left[i_{B \alpha}\right]\right)\left[\boldsymbol{Q}_{\alpha}\right]\left([I]+\left[\boldsymbol{Q}_{\alpha}\right]\right)^{-1}} \\
{\left[\boldsymbol{i}_{A q \beta}\right]=\left(\left[i_{A \beta}\right]-\left[i_{B \beta}\right]\right)\left[\boldsymbol{Q}_{\beta}\right]\left([I]+\left[\boldsymbol{Q}_{\beta}\right]\right)^{-1}} \\
{\left[\boldsymbol{i}_{A q 0}\right]=\left(\left[i_{A 0}\right]-\left[i_{B 0}\right]\right)\left[\boldsymbol{Q}_{0}\right]\left([I]+\left[\boldsymbol{Q}_{0}\right]\right)^{-1}}
\end{gathered}
$$

From the relationships expressed in (45)-(47) and (48)-(50), the current vectors $\left[\boldsymbol{i}_{\lambda \alpha}\right],\left[\boldsymbol{i}_{\lambda \beta}\right]$ and $\left[\boldsymbol{i}_{\lambda 0}\right]$ can be restructured as follows

$$
\begin{gathered}
{\left[\boldsymbol{i}_{\lambda \alpha}\right]=\left[i_{B \alpha}\right]+\left(\left[i_{A \alpha}\right]-\left[i_{B \alpha}\right]\right)\left([I]+\left[\boldsymbol{Q}_{\alpha}\right]\right)^{-1}} \\
{\left[\boldsymbol{i}_{\lambda \beta}\right]=\left[i_{B \beta}\right]+\left(\left[i_{A \beta}\right]-\left[i_{B \beta}\right]\right)\left([I]+\left[\boldsymbol{Q}_{\beta}\right]\right)^{-1}} \\
{\left[\boldsymbol{i}_{\lambda 0}\right]=\left[i_{B 0}\right]+\left(\left[i_{A 0}\right]-\left[i_{B \beta}\right]\right)\left([I]+\left[\boldsymbol{Q}_{\beta}\right]\right)^{-1}}
\end{gathered}
$$

From the previous knowledge of the modal voltages and currents at terminals $A$ and $B$ of each propagation mode, obtained from (17) to (20), currents $i_{A q}, i_{B q}$ and $i_{\lambda}$ can be calculated for $\boldsymbol{p}$ measurements and the system of differential equations based on (26), (29) and
(30) can be solved using the least-squares method to obtain the $\boldsymbol{R}$, $\boldsymbol{L}$ and $\boldsymbol{C}$ parameters in the modal domain.

As a further step, the phase-domain matrices $[\boldsymbol{R}],[\boldsymbol{L}]$ and $[\boldsymbol{C}]$ are obtained from the modal-domain matrices $\left[\boldsymbol{R}_{\mathrm{M}}\right],\left[\boldsymbol{L}_{\mathrm{M}}\right]$ and $\left[\boldsymbol{C}_{\mathrm{M}}\right]$, respectively. The modal matrices are converted into the phase domain using the well-known Clarke's matrix [12, 13, 14]. The estimated $[\boldsymbol{R}],[\boldsymbol{L}]$ and $[\boldsymbol{C}]$ matrices are obtained through the following mode-to-phase transformations

$$
\begin{aligned}
& {[\boldsymbol{R}]=[\boldsymbol{T}]^{\mathrm{T}}\left[\boldsymbol{R}_{\mathrm{M}}\right][\boldsymbol{T}]^{-1}} \\
& {[\boldsymbol{L}]=[\boldsymbol{T}]^{\mathrm{T}}\left[\boldsymbol{L}_{\mathrm{M}}\right][\boldsymbol{T}]^{-1}} \\
& {[\boldsymbol{C}]=[\boldsymbol{T}]^{-1}\left[\boldsymbol{C}_{\mathrm{M}}\right][\boldsymbol{T}]}
\end{aligned}
$$

The process of estimation using the modal-domain method can be summarised as the following algorithm:

(a) Calculate $\left[v_{A}\right]_{m},\left[v_{B}\right]_{m},\left[i_{A}\right]_{m}$ and $\left[i_{B}\right]_{m}$ in (6)-(9) from the fault records, where $m=1,2, \ldots, \boldsymbol{p}$ is the index representing the current and voltage samples recorded during the fault;

(b) Calculate the voltages and currents for the propagation modes $\alpha$, $\beta$ and 0 , as described in (17)-(20);

(c) Calculate $\Delta v$ and $i_{\lambda}$ for the propagation modes $\alpha, \beta$ and 0 , as represented by the single $\pi$ circuit depicted in Fig. 2;

(d) Form the systems of differential equations given by (26), (29) and (30) for the propagation modes $\alpha, \beta$ and 0 ;

(e) Solve the systems of differential equations using the least-squares method [4];

(f) $\left[\boldsymbol{R}_{\mathrm{M}}\right],\left[\boldsymbol{L}_{\mathrm{M}}\right]$ and $\left[\boldsymbol{C}_{\mathrm{M}}\right]$ are obtained from the solution of (26), (29) and (30);

(g) $[\boldsymbol{R}],[\boldsymbol{L}]$ and $[\boldsymbol{C}]$ are estimated from the modal transformations expressed in (54)-(56), where $[\boldsymbol{T}]$ is the Clarke's matrix.

\section{Estimation of line parameters by combining the results of the phase- and modal-domain methods}

The estimation methods based on the phase- and modal-domain line models are used to identify the electrical parameters of an asymmetrical transmission line during a single-phase fault. Using the voltage and current values recorded at both line terminals, the two estimation methods are employed to identify $[\boldsymbol{R}],[\boldsymbol{L}]$ and $[\boldsymbol{C}]$.

The geometrical characteristics of the line are shown in Fig. 3.

The tower structure supports a transmission line with a vertical plane of symmetry. The $\boldsymbol{R}, \boldsymbol{L}$ and $\boldsymbol{C}$ parameters given in (45)-(47) indicate that the transmission line is also non-transposed. These values are used to simulate the reference voltages and currents for

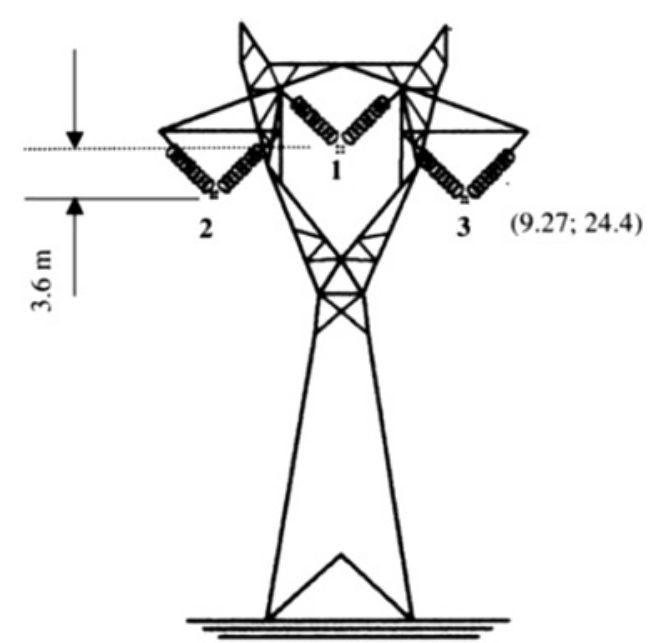

Fig. 3 Transmission line with a vertical plane of symmetry 
the estimation procedure and to calculate the relative errors.

$$
\begin{gathered}
{[\boldsymbol{R}]=\left[\begin{array}{lll}
58.221 & 47.104 & 47.063 \\
47.104 & 58.224 & 47.104 \\
47.063 & 47.104 & 58.221
\end{array}\right] \mathrm{m} \Omega / \mathrm{km}} \\
{[\boldsymbol{L}]=\left[\begin{array}{lll}
1.6876 & 0.8652 & 0.7267 \\
0.8652 & 1.6876 & 0.8652 \\
0.7267 & 0.8652 & 1.6876
\end{array}\right] \mathrm{mH} / \mathrm{km}} \\
{[\boldsymbol{C}]=\left[\begin{array}{ccc}
11.305 & -2.446 & -0.820 \\
-2.446 & 11.775 & -2.446 \\
-0.820 & -2.446 & 11.305
\end{array}\right] \eta \mathrm{F} / \mathrm{km}}
\end{gathered}
$$

An untransposed three-phase line of $300 \mathrm{~km}$ in length was modelled using the software EMTP based on the parameters given in (45)(47). The sending end of the line was connected to a balanced three-phase source of $440 \mathrm{kV}$ at a fundamental frequency of $60 \mathrm{~Hz}$. The receiving end was connected to a three-phase load of $500 \mathrm{kVA}$ and a power factor of 0.97 . A short circuit was simulated at the load terminal of the line in phase 1 , and the fault voltages and fault currents were obtained at both ends simultaneously. The currents obtained for the three phases at the receiving end with a short circuit in phase 1 are shown in Fig. 4.

The duration of the simulation was $0<t<0.3 \mathrm{~s}$. However, the time window shown in Fig. 4 is from 0.1 to $0.3 \mathrm{~s}$ to eliminate the transients following the occurrence of the short circuit. If the high-frequency oscillations in the transient state were to be included, digital signal-processing techniques should be applied to model the voltage and current signals, as in [4].

The same $\boldsymbol{R}, \boldsymbol{L}$ and $\boldsymbol{C}$ parameters given in (57)-(59) are considered as reference values for measuring the relative errors between the calculated and estimated values of $[\boldsymbol{R}],[\boldsymbol{L}]$ and $[\boldsymbol{C}]$.

The relative errors between the calculated and estimated resistances in the matrix $[R]$ that are obtained using both estimation methods are shown in Fig. 5.

The estimation method based on the phase-domain line model appears to be accurate in identifying only the self-resistance of the faulty phase, achieving a relative error of less than $6.5 \%$. The other $\boldsymbol{R}$ parameters that were identified using the phase-domain method exhibit significant errors (greater than 50\%); therefore, these results are discarded and are not shown in Fig. 5. By contrast, the errors in the estimates of the self- and mutual terms in $[\boldsymbol{R}]$ that are obtained using the modal-domain method are acceptable, with relative errors of no greater than $6.5 \%$.

The results obtained for $[\boldsymbol{L}]$ and $[\boldsymbol{C}]$ exhibit behaviour similar to that of those for $[\boldsymbol{R}]$, as shown in Figs. 6 and 7, respectively. The self- and mutual terms in $[\boldsymbol{L}]$ and $[\boldsymbol{C}]$ can be determined with acceptable accuracy using the modal-domain method. As in the case of the resistance, however, the phase-domain method accurately estimates only the self-parameters of the faulty phase. The other self-parameters and the mutual parameters exhibit relative errors greater than $50 \%$ and are excluded.

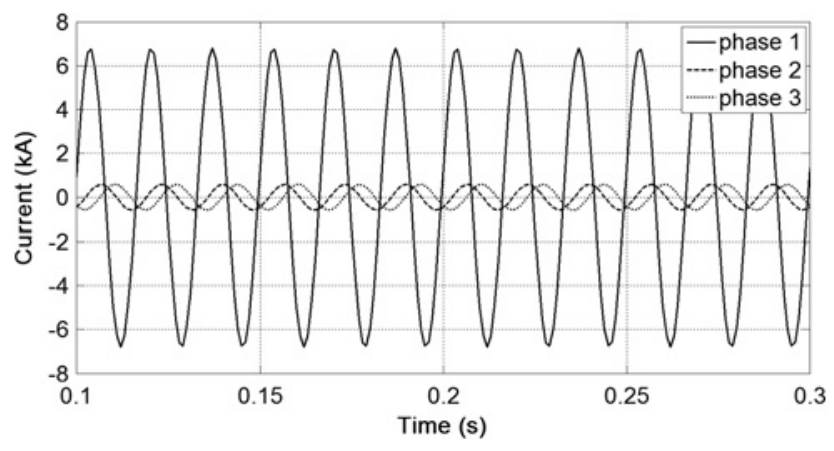

Fig. 4 Short-circuit currents in phases 1, 2 and 3 at the load terminal (receiving end)

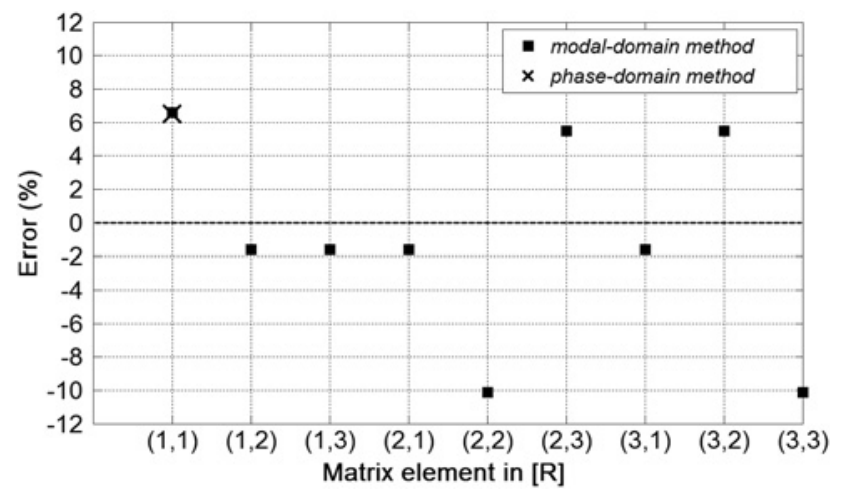

Fig. 5 Relative errors of the self- and mutual $\boldsymbol{R}$ parameters estimated using the modal-and phase-domain methods

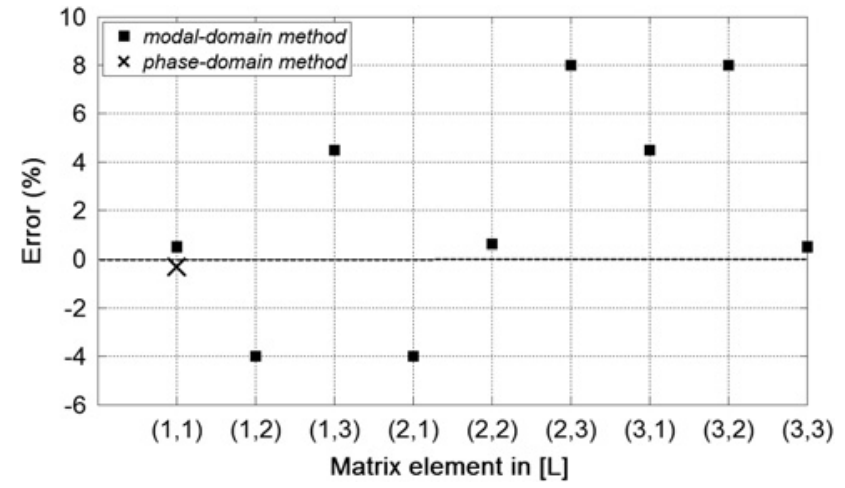

Fig. 6 Relative errors of the self-and mutual $\mathbf{L}$ parameters estimated using the modal-and phase-domain methods

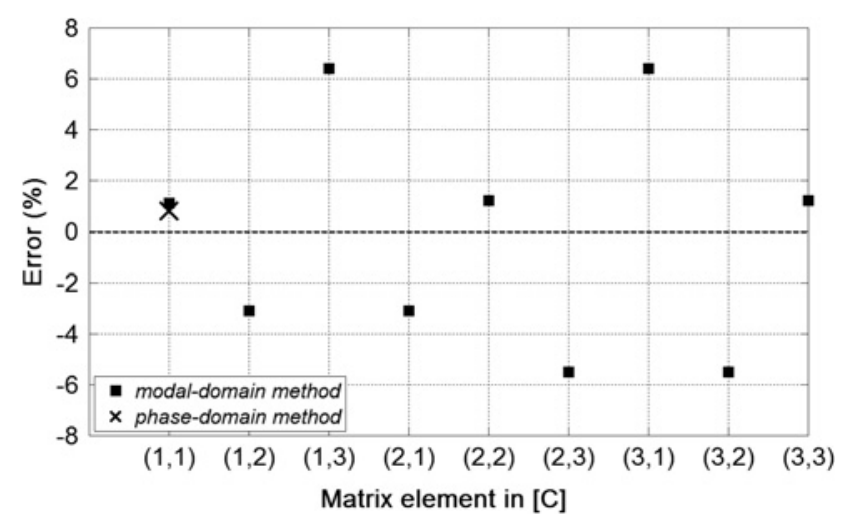

Fig. 7 Relative errors of the self-and mutual $C$ parameters estimated using the modal-and phase-domain methods

An error of approximately $8 \%$ is observed in the mutual inductance between phases 2 and 3, whereas the other mutual and self-terms in $[\underline{\boldsymbol{L}}]$ exhibit relative errors of less than $4.5 \%$. The relative errors of the self- and mutual terms in the capacitance matrix $[\boldsymbol{C}]$ are less than $6.5 \%$.

\section{Discussion of combined methods for parameter determination: performance, reliability and possible applications}

Most methods for parameter determination have limitations, and their performance depends on the characteristics and operating conditions of the transmission system. The accuracy of time- and 
frequency-domain methods depends on factors such as the geometrical/structural characteristics of the line, the load, the operating conditions, the transient conditions, and the use of synchronous or asynchronous measurements at the terminals [3-8].

The novel contribution of this research is the development of a new modal-domain estimation method in the time domain combined with another well-established estimation method that does not require signal modelling using digital signal-processing techniques. The proposed estimation procedure could also be performed to combine two or more estimation methods to increase the reliability of the results.

In this research, two methods based on time-domain line models and measurements are applied. The first method is developed directly in the phase domain using explicit models of the self- and mutual parameters. The second estimation method is developed based on a modal-domain representation of the line, where the propagation modes are decoupled using transformation matrices, thereby eliminating the explicit modelling of the mutual parameters of the three-phase transmission lines, as described in Section 3.

The two estimation methods use voltage and current measurements recorded at the sending and receiving ends of the line during a fault. The phase-domain method provides accurate results only for the self-parameters of the faulty phase, whereas the modal-domain method produces acceptable results for most of the self- and mutual parameters. However, the objective of this study is not to compare the performance of the two estimation methods but rather to determine whether the combination of results obtained from both methods can improve the reliability and accuracy of the parameter estimation.

This section presents a discussion of the estimates of the $[\boldsymbol{R}],[\boldsymbol{L}]$ and $[\boldsymbol{C}]$ matrices obtained using the phase- and modal-domain methods. The results obtained from the two estimation methods are examined in detail, and methods of combining the results of multiple estimation methods to improve the accuracy and reliability of the estimation process are discussed.

\subsection{Resistance matrix}

Among the results presented in Fig. 5, certain values of the mutual resistances estimated using the modal-domain method have errors of less than $6 \%$, whereas most of the values of the mutual resistances have errors of no greater than $2 \%$. By contrast, the errors in the self-resistance are as high as $10 \%$ for phases 2 and 3 and approximately $6 \%$ for phase 1 . The estimation of the self-resistance for phase 1 obtained using the phase-domain method also results in an error close to $6 \%$, nearly identical to that calculated using the modal-domain method. Thus, the estimated resistance value for phase 1 is highly reliable because both methods produce similar results. In addition, although an asymmetrical transmission line was used as the reference, the self-resistance parameters in (45) are very similar. As of this similarity, the same resistance value and relative error obtained for $R(1,1)$ can be extended to $R(2,2)$ and $R(3,3)$ to correct the errors in the estimates of the self-resistances of phases 2 and 3 by approximately $4 \%$, i.e. the same resistance value estimated for $R$ $(1,1)$ can be used for $R(2,2)$ and $R(3,3)$, thereby reducing the relative error from 10 to $6 \%$. The correction procedure, which combines the results estimated using the two methods, is graphically illustrated in Fig. 8 (based on the results previously presented in Fig. 5).

Most of the estimation methods that are available in the technical literature apply to transposed transmission lines. In fact, most transmission lines are transposed, and this characteristic facilitates the estimation process because all of the self-parameters and mutual parameters are similar. Thus, the correction procedure presented in Fig. 8 can be extended to the mutual parameters, i.e. the off-diagonal terms in $[\boldsymbol{Z}]$ and $[\boldsymbol{Y}]$. For example, the resistance value of $R(3,1)$, which has a relative error of less than $2 \%$, can be used as a reference to correct the estimated values of $R(2,3)$ and $R$ $(3,2)$ (approximately 6\%).

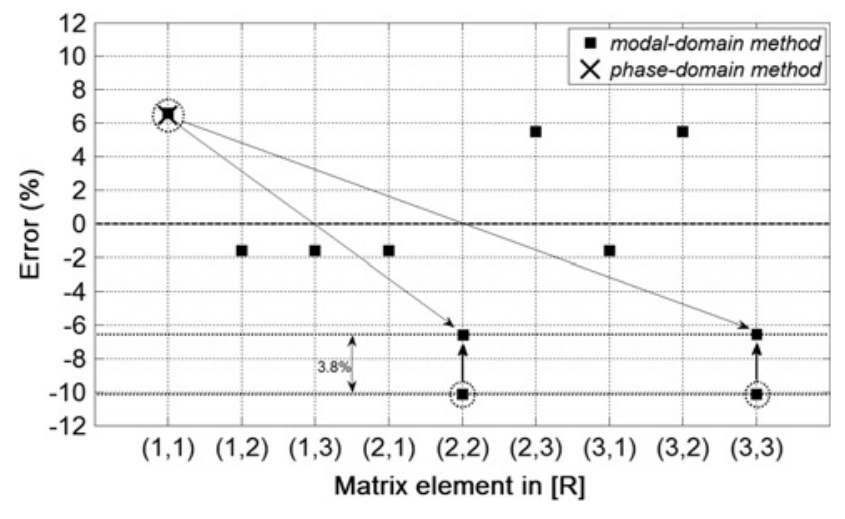

Fig. 8 Fitting (correction) of the $\boldsymbol{R}$ self-parameters by combining the estimates from the phase- and modal-domain methods

A further significant improvement in the reliability of the estimated values could be achieved by using one or two additional estimation methods in parallel with the phase- and modal-domain methods. Estimates obtained using three or more different estimation methods could provide greater redundancy in the results, thereby providing a more accurate and reliable parameter estimation.

\subsection{Inductance and capacitance matrices}

Unlike the resistance matrix $[\boldsymbol{R}]$, the matrices $[\boldsymbol{L}]$ and $[\boldsymbol{C}]$ exhibit low relative errors in their self-terms. The self- $\boldsymbol{L}$ and self- $\boldsymbol{C}$ terms have relative errors of no greater than $1 \%$. In this case, the self-parameters estimated using the phase-domain method improve the reliability of the self-parameters estimated using the modal-domain method because both results produce similar values with very low relative errors.

In Fig. 6, a maximum relative error of $8 \%$ can be observed for the elements $L(2,3)$ and $L(3,2)$, which are, in fact, the same (the mutual inductance between phases 2 and 3). The other mutual inductances have relative errors of no greater than $4.5 \%$. The self-inductances have relative errors of less than $1 \%$, which can be confirmed based on the self-inductance obtained using the phase-domain method for a fault in phase 1 . The difference in the self-inductances estimated using the modal- and phase-domain methods is approximately $0.5 \%$, proving that the estimates of the self-inductance are reliable.

Similar conclusions may be drawn from the results obtained for the capacitance matrix $[\boldsymbol{C}]$. The mutual capacitances have errors of less than $7 \%$, whereas the self-capacitances have errors of no greater than $1 \%$. The self-capacitances estimated using both methods are of very similar magnitudes, as shown in Fig. 7 for a fault in phase 1. This result indicates that the comparison of the two methods that can be applied for phase 1 can also be reasonably extended to phases 2 and 3 , as discussed previously.

\section{Conclusion}

The proposed technique is based on the premise that the combination of the results obtained using two or more estimation methods will improve the reliability of the values of $[\boldsymbol{R}],[\boldsymbol{L}]$ and $[\boldsymbol{C}]$ estimated for multiconductor transmission lines. In this preliminary study, the results obtained from two different estimation methods were combined to improve the accuracy and reliability of the estimated parameters. The first method was developed directly in the phase domain, i.e. the line was modelled directly from the line parameters contained in $[\boldsymbol{Z}]$ and $[\boldsymbol{Y}]$. The second estimation method was developed in the modal domain, i.e. the three-phase line was decoupled into three independent propagation modes, where each mode was modelled as a single-phase line. Both methods were applied based on synchronised measurements of the 
currents and the voltages recorded at the sending and receiving ends of the line during a single-phase fault, specifically, a short circuit at the receiving end in phase 1 .

The modal-domain estimation method produced acceptable results for the self- and mutual parameters of the matrices $[\boldsymbol{R}],[\boldsymbol{L}]$ and $[\boldsymbol{C}]$, whereas the phase-domain method produced accurate results only for the self-parameters of the faulty phase. It was observed that two major benefits could be achieved by comparing the results obtained using the two methods. The first benefit is the improvement in the accuracy of the estimates of the self-terms of the resistance matrix $[\boldsymbol{R}]$ obtained using the modal-domain method. As discussed in Section 5.1, the results obtained using the modal- and phase-domain methods are also useful for improving the accuracy of the estimates of the self-resistances of phases 2 and 3, even for asymmetrical transmission lines. The second benefit of combining the results of the two methods is an increase in reliability, which is achieved because the parameters are estimated using two distinct and independent estimation processes. For example, the values of the self-terms of $[\boldsymbol{L}]$ and $[\boldsymbol{C}]$ that were estimated using the phase- and modal-domain methods exhibited low relative errors and very similar values. These similarities indicated that the estimated values were correct because they were obtained using different and independent estimation methods. These are the major advantages of using multiple estimation methods in parallel for the determination of transmission line parameters. Moreover, the same estimation procedure could also be performed using more than two independent and different estimation methods available in the technical literature to provide greater redundancy.

The combined procedure using both a new modal-domain method and a well-known phase-domain method that is documented in the technical literature represents a simple and efficacious methodology for determining the electrical parameters of transmission lines based on time-domain currents and voltages recorded during a fault. Other, similar techniques could also be developed based on the modal-domain method and other well-established methods in the technical literature. The use of the modal-domain method combined with two or three other estimation methods represents an avenue for further research. Combining the results obtained from two or more different estimation methods could represent an efficient method of obtaining even more reliable and accurate values for $[\boldsymbol{R}],[\boldsymbol{L}]$ and $[\boldsymbol{C}]$ than those obtained using only the two estimation methods investigated in this research. This concept can be explored in further research on estimation procedures based on the same premise followed in this paper: estimation procedures using multiple methods for greater redundancy in the estimated data.

\section{Acknowledgment}

This research was supported by the Fundação de Amparo à Pesquisa do Estado de São Paulo - FAPESP (Proc. 13/00974-5).

\section{References}

1 Hofmman, L: 'Series expansions for line series impedances considering different specific resistances, magnetic permeabilities and dielectric permittivities of conductors, air, and ground', IEEE Trans. Power Deliv., 2003, 18, (2), pp. 564-570

2 Monteiro, J.H.A., Costa, E.C.M., Kurokawa, S., et al.: 'Simplified skin-effect formulation for power transmission lines', IET Sci. Meas. Technol., 2014, 8, pp. $47-53$

3 Zivanovic, R.: 'Estimation of transmission line parameters using fault records'. AUPEC, Victoria University, Melbourne, Australia, 2006, pp. 10-13

4 Schultze, R., Schegner, P., Zivanovic, R.: 'Parameter identification of unsymmetrical transmission lines using fault records obtained from protective relays', IEEE Trans. Power Deliv., 2011, 26, (2), pp. 1265-1272

5 Kurokawa, S., Asti, G.A., Costa, E.C.M., et al.: 'Simplified procedure to estimate the resistance parameters of transmission lines', Electr. Eng., 2013, 95, (3), pp. 221-227

$6 \mathrm{Hu}, \mathrm{Z}$. , Chen, Y.: 'New method of live line measuring the inductance parameters of transmission lines based on GPS technology', IEEE Trans. Power Deliv., 2003, 23 (3), pp. 1288-1295

7 Indulkar, C.S., Ramalingam, K.: 'Estimation of transmission line parameters from measurements', Int. J. Electr. Power Energy Syst., 2008, 30, (5), pp. 337-342

8 Schulze, R., Schegner, P., Stachel, P.: 'Parameter identification of unsymmetrical transmission lines using accurately re-synchronised fault records'. IEEE-PES, Calgary, AB, Canada, 2009, pp. 26-30

9 Rachidi, F., Tkachenko, S.V.: 'Electromagnetic field interaction with transmission lines: from classical theory to HF radiation effects' (WIT Press, 2008)

10 Kurokawa, S., Costa, E.C.M., Pinto, A.J.G., et al.: "Analysis of electric characteristics of high-elevation stretches of the Tucuruí-Macapá-Manaus connection', J. Control Autom. Electr. Syst., 2013, 24, pp. 714-724

11 Dommel, H.W.: 'Electromagnetic transient program reference manual (EMTP theory book)' (Department Electrical Engineering, University of British Columbia, Vancouver, Canada, 1989)

12 Costa, E.C.M., Kurokawa, S., Pissolato, J., et al.: 'Efficient procedure to evaluate electromagnetic transients on three-phase transmission lines', IET Gener. Transm. Distrib., 2010, 4, pp. 1069-1081

13 Costa, E.C.M., Kurokawa, S., Pinto, A.J.G., et al.: 'Simplified computational routine to correct the modal decoupling in transmission lines and power systems modeling', IET Sci. Meas. Technol., 2013, 7, pp. 7-15

14 Paul, C.R.: 'Decoupling the multiconductor transmission line equations', IEEE Trans. Microw. Theory Tech., 1996, 44, (8), pp. 1429-1440 